\title{
Homoclinic Points and Homoclinic Orbits for the Quadratic Family of Real Functions with Two Parameters
}

\author{
Karam N. Abdul-Kareem, Salma M. Farris \\ Department of Mathematics, College of Computer and Mathematical Sciences, University of Mosul, Mosul, Iraq \\ Email: Karamck13s4@gmail.com, Salmaalfaris925@gmail.com
}

How to cite this paper: Abdul-Kareem, K.N. and Farris, S.M. (2020) Homoclinic Points and Homoclinic Orbits for the Quadratic Family of Real Functions with Two Parameters. Open Access Library Journal, 7: e6170.

https://doi.org/10.4236/oalib.1106170

Received: February 16, 2020

Accepted: April 17, 2020

Published: April 20, 2020

Copyright (๑) 2020 by author(s) and Open Access Library Inc.

This work is licensed under the Creative

Commons Attribution International

License (CC BY 4.0).

http://creativecommons.org/licenses/by/4.0/

\section{Abstract}

In this work, we study the homoclinic points and homoclinic orbits of the family of real functions with two parameters $H=\left\{h_{a, b}(x)=a x^{2}+b: a>0, b \in \mathbb{R}\right\}$. We show that the function $h_{a, b} \in H$ has no homoclinic points for $b>\frac{-2}{a}$, but has a homoclinic point for $b \leq \frac{-2}{a}$. Also, we prove that $h_{a, b} \in H$ has homoclinic orbits for $b \leq \frac{-2}{a}$.

\section{Subject Areas}

Dynamical Systems

\section{Keywords}

Local Unstable Set, Unstable Set, Homoclinic Point, Homoclinic Orbit

\section{Introduction}

Recently, we witness that there is a clear attention in connecting orbits (homoclinic or hetroclinic orbits) in dynamical systems [1]. The notion of a homoclinic point was first introduced by ponicaré (1890) [2], in the study of a three-body problem. After about 70 years, Smale $(1963,1976)$ [3], presented the horseshoe notion to show the chaotic behavior of higher-dimensional systems with a transverse homoclinic point. Homoclinic points have been used to study the dynamics of two- or higher-dimensional dynamical systems. Devaney (1989) in [4], showed that the same result holds for a one-dimensional map of an interval into itself with a nondegenerate homoclinic point. Block and Coppel (1992) [5], 
proved that for one-dimensional map $f$ of an interval into itself, a homoclinic point leads to horseshoe for $f^{2}$. Recently, Li (2002) gave a simple proof of Block and Coppel result proof. The concept of homoclinic orbits and heteroclinic connections plays a central role in the studying the chaotic sets. In particular, it can be used for proof of the existence of chaos. In fact, it is proved in [6] [7] [8], the existence of a non-degenerate homoclinic orbit to an expanding fixed point of a smooth map $f$ implies the existence of an invariant set in a neighborhood of the homoclinic orbit, on which $f$ is chaotic. The same result is also true for non-degenerate heteroclinic connections. Homoclinic orbits and heteroclinic connections are relevant not only for the proof of the existence of chaos but also for the description of several bifurcations of chaotic attractors (crisis bifurcations) [9]. In this work, we find homoclinic points and homoclinic orbits for two-parameters family of real functions $H=\left\{h_{a, b}(x)=a x^{2}+b: a>0, b \in \mathbb{R}\right\}$. In Section 2, we study the fixed points of the family

$H=\left\{h_{a, b}(x)=a x^{2}+b: a>0, b \in \mathbb{R}\right\}$ where $h_{a, b}: \mathbb{R} \rightarrow \mathbb{R}$, and the nature of this fixed point for various values $a$ and $b$. In Section 3, we study the local unstable sets of the repelling fixed point $P_{1}$ for the functions $h_{a, b}(x) \in H$. We proved that $w_{l o c}^{u}\left(P_{1}\right)=\left(\frac{1}{2 a}, \infty\right)$. And in Section 4 , we study the unstable sets of the repelling fixed points $P_{1}$ for the functions $h_{a, b}(x) \in H$ we proved that $w^{u}\left(P_{1}\right)=\left(\frac{1}{a}-P_{1}, \infty\right)$. Finally, in Section 5 , we study the homoclinic points and homoclinic orbits of the function $h_{a, b}(x) \in H$ for the repelling fixed point $P_{1}$. We show that $h_{a, b}(x) \in H$ has a homoclinic point and orbit whenever $b \leq \frac{-2}{a}$, and has no homoclinic point and orbit whenever $b>\frac{-2}{a}$. We need some important definitions.

Definition 1: Let $P$ be a repelling fixed point for a map $f: I \rightarrow I$ on a compact interval $I \subset R$. Then the unstable set of $P$ is defined as $w^{u}(P)=\left\{x \in I: \lim _{n \rightarrow \infty} f^{-n}(x)=P\right\} \quad[10]$, which equivalent to the expansivity $|f(x)-f(P)|>|x-P|, \forall x \in I, x \neq P \quad[4]$.

Definition 2: Let $P$ be a repelling fixed point for a map $f: I \rightarrow I$ on a compact interval $I \subset R$ and $U$ be an open interval near $P$. Then, the local unstable set $w_{l o c}^{u}(P)$ of $P$ is defined as $w_{l o c}^{u}(P)=\left\{x \in U: \lim _{n \rightarrow \infty} f^{-n}(x)=P\right\} \quad$ [10]. Which equivalent to the expansivity $|f(x)-f(P)|>|x-P|, \forall x \in U, x \neq P$ [11], and equivalent to $\left|\frac{\mathrm{d} f}{\mathrm{~d} x}\right|>1$ for any $x \in U$ [6].

Definition 3: Let $P$ is fixed point and $f^{\prime}(P)>1$ for a map $f: I \rightarrow I$ on a compact interval $I \subset R$. A point $q$ is called homoclinic pint to $P$ if $q \in w_{l o c}^{u}(P)$ and there exists $n>0$ such that $f^{n}(q)=P$ [4].

Note that the sequence of images of a homoclinic point $q$ and a suitable sequence of preimages of $q$ consist of points which are also homoclinic, and both these sequences converge to $P$. The union of these sequences 
$O(P)=\left\{\cdots, q_{-2}, q_{-1}, q_{0}, q_{1}, q_{2}, \cdots, q_{m}\right\} \quad$ where $\quad q_{0}=q, q_{i+1}=f\left(q_{i}\right)$ for $i \leq m-1$, $q_{m}=P$ and $\lim _{i \rightarrow-\infty} q_{i}=P$, is called the homoclinic orbit of $P[12]$.

\section{The Fixed Points of the Function $h_{a, b}(x) \in H$ and Their Nature}

In this section we study the fixed points of the family $H=\left\{h_{a, b}(x)=a x^{2}+b: a>0, b \in \mathbb{R}\right\}$ where $h_{a, b}: \mathbb{R} \rightarrow \mathbb{R}$, and the nature of these fixed points for various values $a$ and $b$. It is clear that the fixed points of $h_{a, b}(x)$ are $P_{1}=\frac{1+\sqrt{1-4 a b}}{2 a}$ and $P_{2}=\frac{1-\sqrt{1-4 a b}}{2 a}$. The graphs show that the function $h_{a, b}(x)$ has no fixed point for $b>\frac{1}{4 a}$, has a unique indifferent fixed point for $b=\frac{1}{4 a}$ and has two fixed points for $b<\frac{1}{4 a}$. See Figure 1 .

The fixed point $P_{1}$ is always repelling for $b<\frac{1}{4 a}$. But the fixed point $P_{2}$ is attracting for $\frac{-3}{4 a}<b<\frac{1}{4 a}$, indifferent for $b=\frac{-3}{4 a}$ and is repelling for $b<\frac{-3}{4 a}$. See Figure 2.

We need the following remarks in our work.

\subsection{Remark}

For $b<\frac{1}{4 a}, h_{a, b}^{\prime}\left(P_{1}\right)>1$ and for $b<\frac{-3}{4 a}, h_{a, b}^{\prime}\left(P_{2}\right)<-1$.

\section{Proof:}

It is clear that $h_{a, b}^{\prime}(x)=2 a x$, so for $P_{1}=\frac{1+\sqrt{1-4 a b}}{2 a}$,

$h_{a, b}^{\prime}\left(P_{1}\right)=1+\sqrt{1-4 a b}$. If $b<\frac{1}{4 a}$, then $\sqrt{1-4 a b}>0$, is defined and

$h_{a, b}^{\prime}\left(P_{1}\right)=1+\sqrt{1-4 a b}>1$. Now for the fixed point $P_{2}=\frac{1-\sqrt{1-4 a b}}{2 a}$,

$h_{a, b}^{\prime}\left(P_{2}\right)=1-\sqrt{1-4 a b}$. If $b<\frac{-3}{4 a}$, then $\sqrt{1-4 a b}>2$, thus $-\sqrt{1-4 a b}<-2$, there for $h_{a, b}^{\prime}\left(P_{2}\right)=1-\sqrt{1-4 a b}<-1$

According to the definition of the homoclinic points, we consider the repelling fixed point $P_{1}$ and we omit the repelling fixed point $P_{2}$.

\subsection{Remark}

For $h_{a, b}(x) \in H$ with $b<\frac{1}{4 a}$, the fixed point $P_{1}=\frac{1+\sqrt{1-4 a b}}{2 a}>\frac{1}{2 a}$.

Proof:

Let $b<\frac{1}{4 a}$. Then $\sqrt{1-4 a b}>0$ is defined and thus, $1+\sqrt{1-4 a b}>1$. There for $P_{1}=\frac{1+\sqrt{1-4 a b}}{2 a}>\frac{1}{2 a}$ 


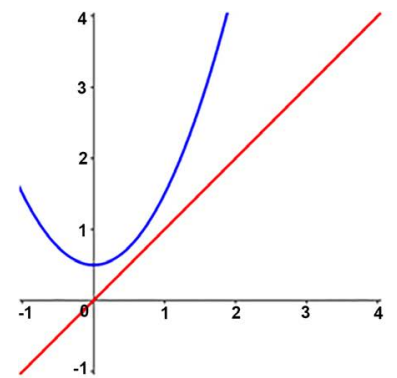

(a)

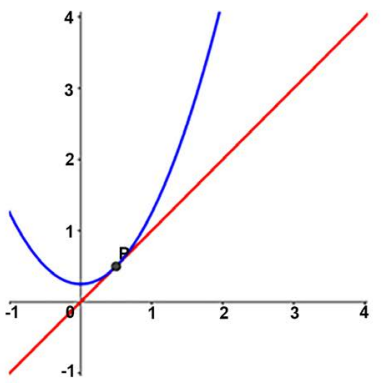

(b)

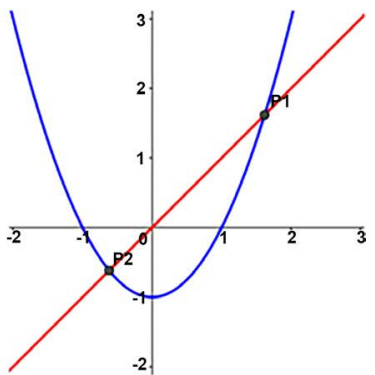

(c)

Figure 1. (a) $a=1, b=0.5$; (b) $a=1, b=-0.25$; (c) $a=1, b=-1$.

\section{$P_{1}$ is repelling}

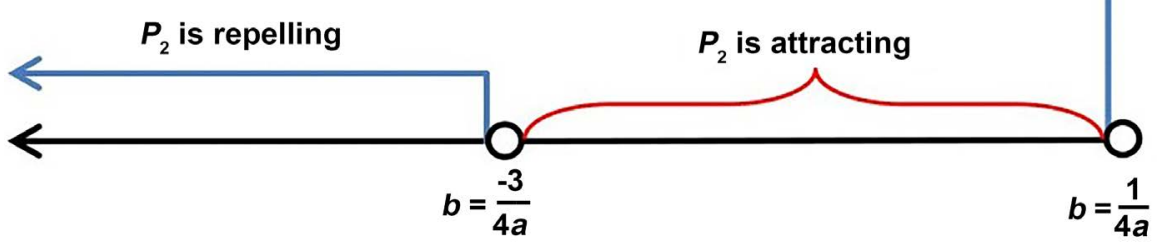

Figure 2. Nature of $P_{1}$ and $P_{2}$.

\section{The Local Unstable Sets}

In this section, we study the local unstable sets of the repelling fixed point $P_{1}$ for the functions $h_{a, b}(x) \in H$.

\section{Proposition}

For $h_{a, b}(x) \in H$ the local unstable set of the fixed point $P_{1}$ is $w_{l o c}^{u}\left(P_{1}\right)=\left(\frac{1}{2 a}, \infty\right)$.

\section{Proof:}

To find the local unstable for the repelling fixed point $P_{1}$ we consider the inequality $\left|h_{a, b}^{\prime}\left(P_{1}\right)\right|>1$ [6]. Since $h_{a, b}$ is a continuous function, then there exists a neighborhood $U$ of the fixed point $P_{1}$ such that $\left|h_{a, b}^{\prime}(x)\right|>1, \forall x \in U$, i.e. $|2 a x|>1, \forall x \in U$. Thus $|x|>\frac{1}{2|a|}=\frac{1}{2 a}$. Then either $x<\frac{-1}{2 a}$, i.e. $x \in\left(-\infty, \frac{-1}{2 a}\right)$, or $x>\frac{1}{2 a}$, i.e. $x \in\left(\frac{1}{2 a}, \infty\right)$. By remark (2.2) $P_{1}>\frac{1}{2 a}$, there for $w_{l o c}^{u}\left(P_{1}\right)=\left(\frac{1}{2 a}, \infty\right)$.

\section{The Unstable Sets}

The unstable sets of the repelling fixed points $P_{1}$ for the functions $h_{a, b}(x) \in H$ is calculated in the following proposition.

We need the following lemma in our studying. 


\subsection{Lemma}

For $h_{a, b}(x) \in H, h_{a, b}^{-1}\left(P_{1}\right)=\mp \sqrt{\frac{P_{1}-b}{a}}=\mp P_{1}$ where $P_{1}>b$.

The proof is clear.

\subsection{Proposition}

For $h_{a, b}(x) \in H$ the unstableset of the fixed point $P_{1}$ is $w^{u}\left(P_{1}\right)=\left(\frac{1}{a}-P_{1}, \infty\right)$.

\section{Proof:}

Consider the expansive inequality $\left|h_{a, b}(x)-P_{1}\right|>\left|x-P_{1}\right|$ where $x \neq P_{1}$.

Then for $h_{a, b}(x) \in H$, we have $\left|a x^{2}+b-P_{1}\right|>\left|x-P_{1}\right|$, thus $a\left|x-\sqrt{\frac{\left(P_{1}-b\right)}{a}}\right|\left|x+\sqrt{\frac{\left(P_{1}-b\right)}{a}}\right|>\left|x-P_{1}\right|$. By lemma (4.1). Thus we have $a\left|x-P_{1}\right|\left|x+P_{1}\right|>\left|x-P_{1}\right|$. There for $\left|x+P_{1}\right|>\frac{1}{a}$.

Either $x+P_{1}<\frac{-1}{a}$ which implies $x<\frac{-1}{a}-P_{1}$. Or $x+P_{1}>\frac{1}{a}$, which implies $x>\frac{1}{a}-P_{1}$. That is $x \in\left(-\infty, \frac{-1}{a}-P_{1}\right) \cup\left(\frac{1}{a}-P_{1}, \infty\right)$. To calculate $w^{u}\left(P_{1}\right)$, by remark (2.2), $P_{1}>\frac{1}{2 a}>0$. Now $\left(-\infty, \frac{-1}{a}-P_{1}\right) \subset \mathbb{R}^{-}$, which implies $P_{1} \notin\left(-\infty, \frac{-1}{a}-P_{1}\right)$. So $w^{u}\left(P_{1}\right)=\left(\frac{1}{a}-P_{1}, \infty\right)$.

\subsection{Example}

For $h_{1,-6}(x)=x^{2}-6$, then $w^{u}\left(P_{1}\right)=(-2, \infty)$.

\section{Solution:}

It is clear that $P_{1}=3$. Since $\left|h_{1,-6}(x)-P_{1}\right|>\left|x-P_{1}\right|$, where $x \neq P_{1}$, implies that $\left|x^{2}-6-3\right|>|x-3|$, then $\left|x^{2}-9\right|>|x-3|$. Thus $|(x-3)(x+3)|>|x-3|$. Since $x \neq 3$, then $|(x+3)|>1$, there for either $x+3<-1$, then $x<-4$, i.e. $x \in(-\infty,-4)$.

Or $x+3>1$, then $x>-2$, i.e. $x \in(-2, \infty)$. Thus $x \in(-\infty,-4) \cup(-2, \infty)$.

But $P_{1}=3 \in(-2, \infty)$, then $w^{u}\left(P_{1}\right)=\left(\frac{1}{1}-3, \infty\right)$.

\section{Homoclinic Points and Homoclinic Orbits for the Family $\boldsymbol{H}$}

In this section, we study the homoclinic points and homoclinic orbits of the function $h_{a, b}(x) \in H$ for the repelling fixed point $P_{1}=\frac{1+\sqrt{1-4 a b}}{2 a}$ where $b<\frac{1}{4 a}$, note that the other repelling fixed point $P_{2}=\frac{1-\sqrt{1-4 a b}}{2 a}$. By remark (2.1), $h_{a, b}^{\prime}\left(P_{2}\right)<-1$ where $b<\frac{-3}{4 a}$. Thus according to the definition of the homoclinic point, we don't study the homoclinic points of $P_{2}$. 


\subsection{Homoclinic Points for the Functions $h_{a, b}(x) \in H$}

To study the homoclinic points of the repelling fixed point $P_{1}$ of $h_{a, b}(x)$ we use the following technique concerned on the preimages of $h_{a, b}(x)$.

\subsubsection{Remark}

For $h_{a, b}(x) \in H, h_{a, b}^{-2}\left(P_{1}\right)=\mp \sqrt{\frac{-P_{1}-b}{a}}$.

\section{Proof:}

By lemma (4.1), $h_{a, b}^{-1}\left(P_{1}\right)=\mp P_{1}$. Then $h_{a, b}^{-2}\left(P_{1}\right)=h_{a, b}^{-1}\left( \pm P_{1}\right)=\mp \sqrt{\frac{ \pm P_{1}-b}{a}}$. For $h_{a, b}^{-2}\left(P_{1}\right)=h_{a, b}^{-1}\left(+P_{1}\right)=\mp \sqrt{\frac{+P_{1}-b}{a}}= \pm P_{1}$, so we have the same state, so is omitted. For $h_{a, b}^{-2}\left(P_{1}\right)=h_{a, b}^{-1}\left(-P_{1}\right)=\mp \sqrt{\frac{-P_{1}-b}{a}}$.

Now if $-P_{1} \geq b$, in remark (5.1.1), then put $h_{a, b}^{-2}\left(P_{1}\right)=\mp \sqrt{\frac{-P_{1}-b}{a}}=\mp q_{1, j}$ where $j \in N$. For $h_{a, b}^{-1}\left(q_{1, j}\right)=h_{a, b}^{-3}\left(P_{1}\right)=\mp \sqrt{\frac{ \pm q_{1, j}-b}{a}}=\mp q_{2, j}$, where $\pm q_{1, j}>b$. Similarly for $h_{a, b}^{-1}\left( \pm q_{2, j}\right)=h_{a, b}^{-4}\left(P_{1}\right)=\mp \sqrt{\frac{ \pm q_{2, j}-b}{q}}=\mp q_{3, j}$, where $\pm q_{2, j}>b$.

And so on (see Figure 3). Thus we have sequences of preimage points of $h_{a, b}(x), \quad q_{i, j} \in \mathbb{R}$. If $\mp q_{i, j} \in w_{l o c}^{u}\left(P_{1}\right)$, for some $i, j \in N$, then these points are the homoclinic points of the repelling fixed point $P_{1}$.

In following propositions and examples we study the homoclinic points of the fixed point $P_{1}$ of $h_{a, b}(x) \in H$ for various values of $a$ and $b$.

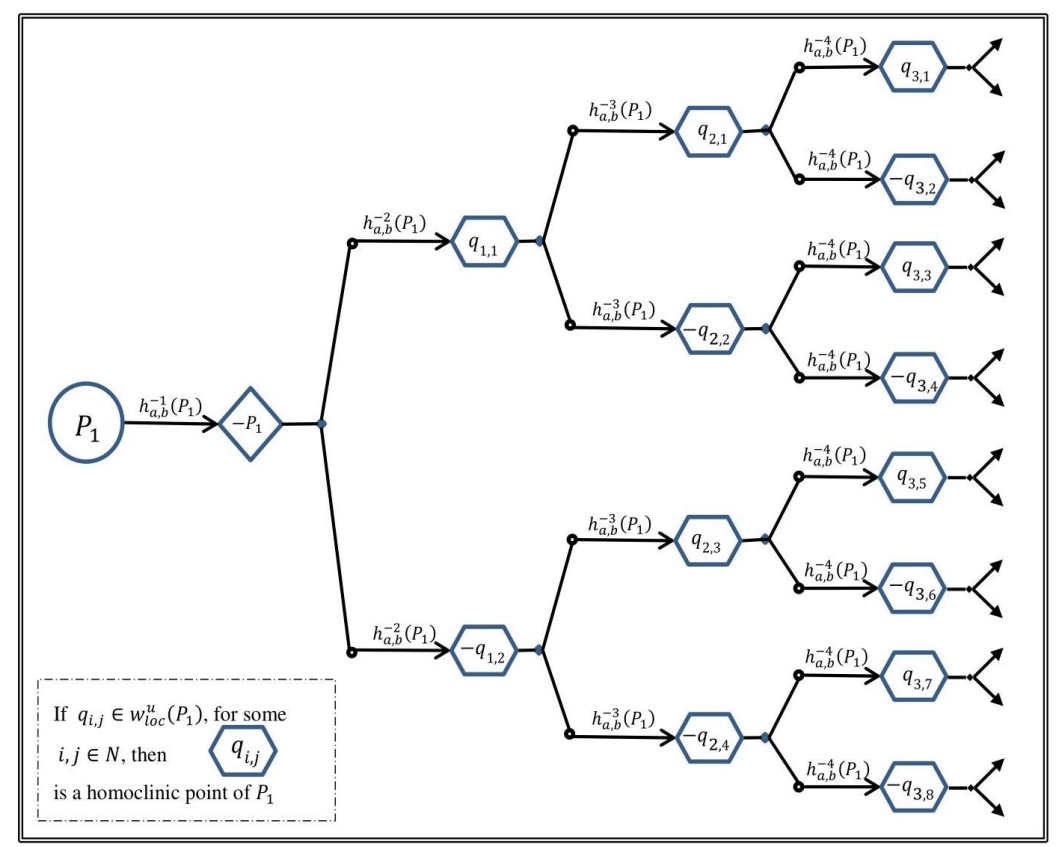

Figure 3. Tree of the homoclinic points of $h_{a, b}(x)$. 


\subsubsection{Proposition}

For $h_{a, b}(x) \in H$. If $b=0$ then the fixed point $P_{1}$ has no homoclinic points.

\section{Proof:}

It is clear that $h_{a, 0}(x)=a x^{2}$ and $P_{1}=\frac{1}{a}$. By proposition (3.1),

$w_{l o c}^{u}\left(P_{1}\right)=\left(\frac{1}{2 a}, \infty\right)$. Now the first preimage of $h_{a, 0}(x)$ is $h_{a, 0}^{-1}(x)=\mp \sqrt{\frac{x}{a}}$

where $x>0$. For $P_{1}=\frac{1}{a}$ and by lemma (4.1), we have

$h_{a, 0}^{-1}\left(\frac{1}{a}\right)=\mp \sqrt{\frac{1}{a^{2}}}=\mp \frac{1}{a}=\mp P_{1}$. But $+P_{1}=\frac{1}{a}$ is a fixed point, and

$-P_{1}=-\frac{1}{a} \notin w_{l o c}^{u}\left(P_{1}\right)=\left(\frac{1}{2 a}, \infty\right)$. Moreover, by proposition (5.1.1), we have

$h_{a, 0}^{-2}\left(P_{1}\right)=\mp \sqrt{\frac{-P_{1}}{a}}= \pm \sqrt{\frac{-1}{a^{2}}} \notin \mathbb{R}$, since $\frac{1}{a^{2}}>0$. There for $h_{a, 0}^{-n}\left(P_{1}\right)$ is undefined in $\mathbb{R}$ for $n \geq 2$. Thus $h_{a, 0}(x)=a x^{2}$ has no homoclinic point to the fixed point $P_{1}$.

\subsubsection{Example}

For $h_{1,0}(x)=x^{2}$ has no homoclinic points to the fixed point $P_{1}=1$.

\section{Solution:}

By proposition (3.1), $w_{l o c}^{u}\left(P_{1}\right)=\left(\frac{1}{2}, \infty\right)$. The first preimage of $h_{1,0}(x)$ is $h_{1,0}^{-1}(x)=\mp \sqrt{x} \quad$ where $x>0$. For $h_{1,0}^{-1}(1)=\mp \sqrt{1}= \pm 1= \pm P_{1}$. But $P_{1}=1$ is a fixed point of $h_{1,0}(x)$, and $-1 \notin w_{\text {loc }}^{u}\left(P_{1}\right)$. Now for $h_{1,0}^{-1}(-1)=h_{1,0}^{-2}(1)=\mp \sqrt{-1} \notin \mathbb{R}$. So $h_{1,0}(x)=x^{2}$ has no homoclinic points to the fixed point $P_{1}$.

\subsubsection{Proposition}

For $h_{a, b}(x) \in H$, the first preimage of the fixed point $P_{1}$ cannot be a homoclinic point to the fixed point $P_{1}$.

\section{Proof:}

The first preimage of $h_{a, b}(x)$ is $h_{a, b}^{-1}(x)=\mp \sqrt{\frac{x-b}{a}}$ where $x>b$. For $P_{1}=\frac{1+\sqrt{1-4 a b}}{2 a}$ and by lemma (4.1) we have $h_{a, b}^{-1}\left(P_{1}\right)=\mp \sqrt{\frac{P_{1}-b}{a}}=\mp P_{1}$ where $P_{1}>b$. But $+P_{1}$ is a fixed point, by remark (2.2) $P_{1}>\frac{1}{2 a}$, we have $-P_{1}<\frac{-1}{2 a}$ and $w_{l o c}^{u}\left(P_{1}\right)=\left(\frac{1}{2 a}, \infty\right)$.

So $-P_{1} \notin w_{l o c}^{u}\left(P_{1}\right)=\left(\frac{1}{2 a}, \infty\right)$. Thus the fixed point $P_{1}$ has no homoclinic points at the first preimage.

\subsubsection{Example}

$h_{1,-2}(x)=x^{2}-2$ has no homoclinic points to the fixed point $P_{1}$ in the first 
preimage.

\section{Solution:}

The fixed point $P_{1}=2$, and the first preimage of $h_{1,-2}(x)$ is $h_{1,-2}^{-1}(x)=\mp \sqrt{x+2}$. From proposition (3.1), $w_{\text {loc }}^{u}\left(P_{1}\right)=\left(\frac{1}{2}, \infty\right)$. For $P_{1}=2$ then $h_{1,-2}^{-1}(2)=\mp \sqrt{2+2}= \pm 2= \pm P_{1}$. But $P_{1}=2$ is a fixed point of $h_{1,-2}(x)$ and $-2 \notin w_{\text {loc }}^{u}\left(P_{1}\right)$. So $h_{1,-2}(x)=x^{2}-2$ has no homoclinic points to the fixed point $P_{1}$ in the first preimage.

Following remarkes assert that some inverse images of $P_{1}, h_{a, b}^{-n}\left(P_{1}\right)$ are belong to the local unstable set of $P_{1}$ for $b \leq \frac{-2}{a}$.

\subsubsection{Remark}

If $b<\frac{-(5+2 \sqrt{5})}{4 a}$ of $h_{a, b} \in H$, then the second preimage of the fixed point $P_{1}$ belongs to the local unstable set of $P_{1}$.

\section{Proof:}

Let $b<\frac{-5-2 \sqrt{5}}{4 a}$, it is clearly that $b<\frac{-5+2 \sqrt{5}}{4 a}$. There for $a b+\frac{5+2 \sqrt{5}}{4}<0$ and $a b+\frac{5-2 \sqrt{5}}{4}<0$. So $\left(a b+\frac{5+2 \sqrt{5}}{4}\right)\left(a b+\frac{5-2 \sqrt{5}}{4}\right)>0$.

Then $4 a^{2} b^{2}+10 a b+\frac{5}{4}>0$, thus $1-4 a b<4 a^{2} b^{2}+6 a b+\frac{9}{4}$, which implies $1-4 a b<\left(2 a b+\frac{3}{2}\right)^{2}$. Since $\left(2 a b+\frac{3}{2}\right)^{2}=\left(-\left(2 a b+\frac{3}{2}\right)\right)^{2}$, there for $1-4 a b<\left(-2 a b-\frac{3}{2}\right)^{2}$, then $\sqrt{1-4 a b}<-2 a b-\frac{3}{2}$. Thus $\frac{1+\sqrt{1-4 a b}}{2 a}<-b-\frac{1}{4 a}$, i.e. $P_{1}<-b-\frac{1}{4 a}$. There for $\sqrt{\frac{-P_{1}-b}{a}}>\frac{1}{2 a}$. By remark (5.1.1) $h_{a, b}^{-2}\left(P_{1}\right)=\mp \sqrt{\frac{-P_{1}-b}{a}}$, So $h_{a, b}^{-2}\left(P_{1}\right)>\frac{1}{2 a}$. Thus $h_{a, b}^{-2}\left(P_{1}\right) \in w_{l o c}^{u}\left(P_{1}\right)$. (See proposition (3.1)).

\subsubsection{Remark}

If $\frac{-(5+2 \sqrt{5})}{4 a} \leq b \leq \frac{-2}{a}$ of $h_{a, b} \in H$, then the third preimage of the fixed point $P_{1}$ belongs to the local unstable set of $P_{1}$.

The proof is same of the above remark with some more complicated details.

\subsubsection{Theorem}

For the family $H=\left\{h_{a, b}(x)=a x^{2}+b\right\}$, there exist homoclinic points to the fixed point $P_{1}$ whenever $b \leq \frac{-2}{a}$.

Proof: 
According to a proposition (5.1.4), we begin with the second preimage of the fixed point $P_{1}$. By remark (5.1.1), we have

$$
\begin{gathered}
h_{a, b}^{-2}\left(P_{1}\right)= \pm \sqrt{\frac{-P_{1}-b}{a}} \text {. Suppose that } \pm \sqrt{\frac{-P_{1}-b}{a}} \in \mathbb{R} \text {, i.e. }-P_{1} \geq b \text {, then } \\
P_{1} \leq-b \text {. Since } P_{1}=\frac{1+\sqrt{1-4 a b}}{2 a} \text {, where } b<\frac{1}{4 a} \text {. Then } \frac{1+\sqrt{1-4 a b}}{2 a} \leq-b,
\end{gathered}
$$

which implies

$$
\sqrt{1-4 a b} \leq-2 a b-1 \text {. }
$$

Let $c=-2 a b-1$. Since $b<\frac{1}{4 a}$, then $\sqrt{1-4 a b}>0$, which implies $0<\sqrt{1-4 a b} \leq c$, i.e. $c \in(0, \infty)$

Then we have three cases for $b$

Case 1: If $b>0$, then $a b>0$, thus $-2 a b<0$, there for $-2 a b-1<-1$, which implies $c<-1$ which is a contradiction with $c \in(0, \infty)$ in $\left(^{\star}\right)$.

Case 2: If $b=0$, then by proposition (5.1.2) there is no homoclinic points to the fixed point $P_{1}$.

Case 3: If $b<0$, then $a b<0$. Thus $-2 a b>0$, there for $-2 a b-1>-1$, which implies $c>-1$, i.e. $c \in(-1, \infty)$. Since $c \in(0, \infty)$ in $(*)$, then $c \in(-1, \infty) \cap(0, \infty)=(0, \infty)$. There for $\sqrt{1-4 a b} \leq-2 a b-1$, implies that $1-4 a b \leq 4 a^{2} b^{2}+4 a b+1$, thus $\left.4 a b(a b+2) \geq 0 \ldots \ldots \ldots{ }^{* *}\right)$.

Since $a>0$ and $b<0$, then $4 a b<0$, so by $\left(^{\star *}\right), a b+2 \leq 0$, thus $a b \leq-2$. It follows that $b \leq \frac{-2}{a}$. By remark (2.1), $h_{a, b}^{\prime}\left(P_{1}\right)>1$.

Now:

1) For $b<\frac{-(5+2 \sqrt{5})}{4 a}$, let $h_{a, b}^{-2}\left(P_{1}\right)=q_{1,1}$, by remark (5.1.6), $q_{1,1} \in w_{l o c}^{u}\left(P_{1}\right)$ and it is clear that $h_{a, b}^{2}\left(q_{1,1}\right)=P_{1}$ (see Figure 3).

2) For $\frac{-(5+2 \sqrt{5})}{4 a} \leq b \leq \frac{-2}{a}$, let $h_{a, b}^{-3}\left(P_{1}\right)=q_{2,1}$, by remark (5.1.7), $q_{2,1} \in w_{\text {loc }}^{u}\left(P_{1}\right)$ and it is clear that $h_{a, b}^{3}\left(q_{2,1}\right)=P_{1}$ (see Figure 3 ).

There for $q_{1,2}$ for $\frac{-(5+2 \sqrt{5})}{4 a} \leq b \leq \frac{-2}{a}$ and $q_{1,1}$ for $b<\frac{-(5+2 \sqrt{5})}{4 a}$ are the first homoclinic points for the repelling fixed point $P_{1}$.

\subsubsection{Theorem}

If $b>\frac{-2}{a}$, then $h_{a, b}(x)=a x^{2}+b$ has no homoclinic points to the fixed point $P_{1}$.

\section{Proof:}

According to a proposition (5.1.4), we begin with the second preimage of the fixed point $P_{1}$. By remark (5.1.1), we have $h_{a, b}^{-2}\left(P_{1}\right)= \pm \sqrt{\frac{-P_{1}-b}{a}}$. Since $P_{1}$ is a repelling fixed point for $b<\frac{1}{4 a}$. So, with 
our assumption, we have $\frac{-2}{a}<b<\frac{1}{4 a}$. Now we divide the proof into three cases:

Case 1: If $\frac{-2}{a}<b<0$, then $-2<a b<0$. Thus $0<a b+2<2$, and $-8<4 a b<0$. So $4 a b(a b+2)<0$, which implies $4 a^{2} b^{2}+4 a b+1<1-4 a b$. Thus

$$
(2 a b+1)^{2}<1-4 a b
$$

Since $(2 a b+1)^{2}=(-(2 a b+1))^{2}$ and $\frac{-2}{a}<b<0$, then $1-4 a b>0$. Thus $\left(^{*}\right)$ will being $-2 a b-1<\sqrt{1-4 a b}$, which implies, $-b<\frac{1+\sqrt{1-4 a b}}{2 a}$, i.e. $-b<P_{1}$, which implies $\frac{-P_{1}-b}{a}<0$. So

$$
h_{a, b}^{-2}\left(P_{1}\right)= \pm \sqrt{\frac{-P_{1}-b}{a}} \notin \mathbb{R} \text {. There for } h_{a, b}^{-n}\left(P_{1}\right) \notin \mathbb{R}, \forall n \in N \text {. Then } h_{a, b}(x) \text { has }
$$
no homoclinic points to the fixed point $P_{1}$ for $\frac{-2}{a}<b<0$.

Case 2: If $b=0$, then by proposition (5.1.2) there is no homoclinic point to the fixed point $P_{1}$.

Case 3: If $0<b<\frac{1}{4 a}$, then $0<a b<\frac{1}{4}$. Thus $2<a b+2<\frac{9}{4}$, and $0<4 a b<1$. So $4 a b(a b+2)>0$, which implies $4 a^{2} b^{2}+4 a b+1>1-4 a b$, thus $(2 a b+1)^{2}>1-4 a b$ $\left({ }^{*}\right)$, Since

$(2 a b+1)^{2}=(-(2 a b+1))^{2}$ and $0<b<\frac{1}{4 a}$, then $1-4 a b>0$. Thus $\left(^{*}\right)$ will being $-2 a b-1>\sqrt{1-4 a b}$, which implies, $-b>\frac{1+\sqrt{1-4 a b}}{2 a}$, i.e. $-b>P_{1}$, which implies $\frac{-P_{1}-b}{a}>0$. So $h_{a, b}^{-2}\left(P_{1}\right)= \pm \sqrt{\frac{-P_{1}-b}{a}} \in \mathbb{R}$. But by theorem (5.1.8), if $\pm \sqrt{\frac{-P_{1}-b}{a}} \in \mathbb{R}$, then $b \leq \frac{-2}{a}$ which is a contradiction with $0<b<\frac{1}{4 a}$. There for $h_{a, b}(x)$ has no homoclinic points to the fixed point $P_{1}$ for $0<b<\frac{1}{4 a}$.

Following examples explain the cases for $b<\frac{-2}{a}, b=\frac{-2}{a}$ and $b>\frac{-2}{a}$ respectively.

\subsubsection{Example}

For $h_{2,-3}(x)=2 x^{2}-3$, has a homoclinic point to the fixed point $P_{1}$ at (0.86602540378).

\section{Solution:}

The fixed point $P_{1}=\frac{3}{2}=1.5$ and the first preimage of $h_{2,-3}(x)$ is $h_{2,-3}^{-1}(x)=\mp \sqrt{\frac{x+3}{2}}$. From proposition (3.1), $w_{l o c}^{u}\left(P_{1}\right)=\left(\frac{1}{4}, \infty\right)$. Clearly 
$h_{1,-2}^{\prime}(1.5)>1$. For $P_{1}=1.5$, then $h_{2,-3}^{-1}(1.5)=\mp \sqrt{\frac{1.5+3}{2}}= \pm 1.5= \pm P_{1}$. But $P_{1}=1.5$ is a fixed point of $h_{2,-3}(x)$, and $-1.5 \notin w_{l o c}^{u}\left(P_{1}\right)$. Now for

$$
h_{2,-3}^{-1}(-1.5)=h_{2,-3}^{-2}(1.5)=\mp \sqrt{\frac{-1.5+3}{2}}= \pm 0.86602540378 \text {, }
$$

where

$0.86602540378 \in w_{l o c}^{u}\left(P_{1}\right) \quad$ and $\quad-0.86602540378 \notin w_{l o c}^{u}\left(P_{1}\right)$. Moreover $\mathrm{h}_{2,-3}(0.86602540378)=-1.5 \quad$ and $\quad \mathrm{h}_{1,-2}^{2}(0.86602540378)=1.5 \quad$ So $(0.86602540378)$ is a homoclinic point to the fixed point $P_{1}$.

\subsubsection{Remark}

Here we consider 0.86602540378 (the first) homoclinic point for the fixed point (1.5). In fact, there are many points belong to the local unstable set of 1.5 (i.e. homoclinic points to $\left.P_{1}=1.5\right)$. In fact

$$
\begin{aligned}
& h_{2,-3}^{-1}(0.86602540378)=h_{2,-3}^{-3}(1.5)=\mp \sqrt{\frac{0.86602540378+3}{2}}=\mp 1.390328271 . \\
& \text { Now, } 1.390328271 \in w_{l o c}^{u}\left(P_{1}\right), \text { and }-1.390328271 \notin w_{l o c}^{u}\left(P_{1}\right) . \text { For } \\
& h_{2,-3}^{-1}(-0.86602540378)=h_{2,-3}^{-3}(1.5)=\mp \sqrt{\frac{-0.86602540378+3}{2}}=\mp 1.032950772,
\end{aligned}
$$

and

$1.032950772 \in w_{l o c}^{u}\left(P_{1}\right)$, and $-1.032950772 \notin w_{l o c}^{u}\left(P_{1}\right)$. If we continue with this way, we get a set $\{0.86602540378,1.390328271,1.032950772, \cdots\}$. Every point in this set belongs to $w_{\text {loc }}^{u}\left(P_{1}\right)$. Each point of this set is a homoclinic point to the fixed point $P_{1}=1.5$. See Figure 4 .

\subsubsection{Example}

For $h_{1,-2}(x)=x^{2}-2, \sqrt{2}$ is a homoclinic point to the fixed point $P_{1}$.

\section{Solution:}

It is clear that $P_{1}=2$, and the first preimage of $h_{1,-2}(x)$ is $h_{1,-2}^{-1}(x)=\mp \sqrt{x+2}$.

From proposition (3.1), $w_{l o c}^{u}\left(P_{1}\right)=\left(\frac{1}{2}, \infty\right)$. Clearly $h_{1,-2}^{\prime}(2)>1$. For $P_{1}=2$, then $h_{1,-2}^{-1}(2)=\mp \sqrt{2+2}= \pm 2= \pm P_{1}$. But $P_{1}=2$ is a fixed point of $h_{1,-2}(x)$, and $-2 \notin w_{\text {loc }}^{u}\left(P_{1}\right)$. Now for $h_{1,-2}^{-1}(-2)=h_{1,-2}^{-2}(2)=\mp \sqrt{-2+2}=0 \notin w_{l o c}^{u}\left(P_{1}\right)$. Now for $h_{1,-2}^{-1}(0)=h_{1,-2}^{-3}(2)=\mp \sqrt{0+2}=\mp \sqrt{2}$.

$\sqrt{2} \in w_{\text {loc }}^{u}\left(P_{1}\right)$ and $-\sqrt{2} \notin w_{l o c}^{u}\left(P_{1}\right)$. Moreover $h_{1,-2}(\sqrt{2})=0, h_{1,-2}^{2}(\sqrt{2})=-2$ and $h_{1,-2}^{3}(\sqrt{2})=2$. So $\sqrt{2}$ is a homoclinc point to the fixed point $P_{1}=2$.

In fact, any preimage point contained in $w_{l o c}^{u}\left(P_{1}\right)$ is a homoclinic point for $P_{1}$. See Figure 5.

\subsubsection{Example}

$h_{1,-1}(x)=x^{2}-1$, has no homoclinic points to the fixed point $P_{1}$. 


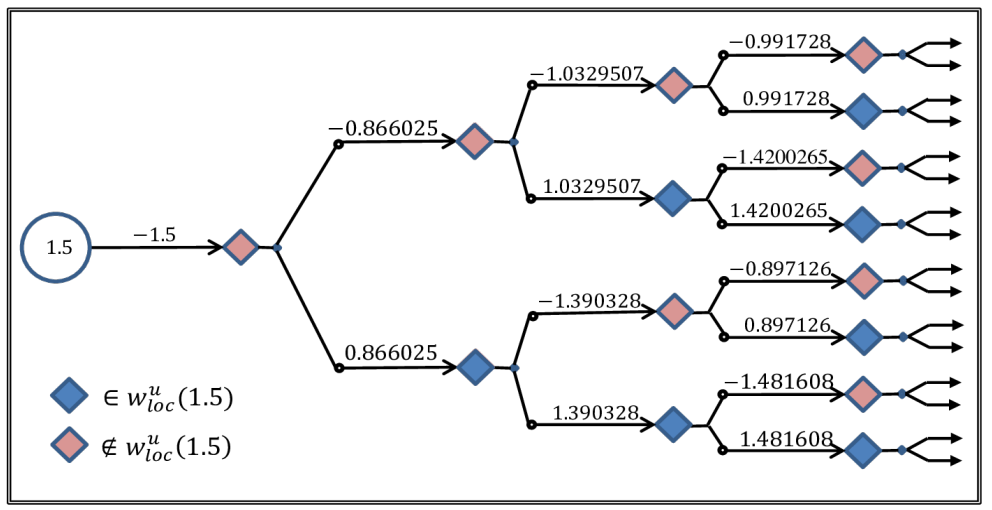

Figure 4. Tree of the homoclinic points of $h_{2,-3}(x)=2 x^{2}-3$.

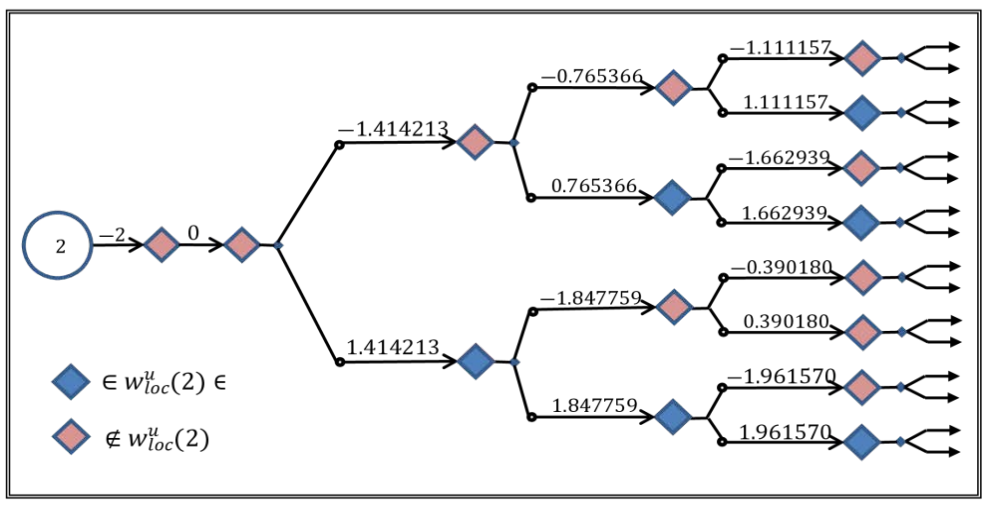

Figure 5. Tree of the homoclinic points of $h_{1,-2}(x)=x^{2}-2$.

\section{Solution:}

It is clear that $P_{1}=\frac{1+\sqrt{5}}{2}=1.618033989$, and the first preimage of $h_{1,0}(x)$ is $h_{1,-1}^{-1}(x)=\mp \sqrt{x+1}$.

From proposition (3.1), $w_{l o c}^{u}\left(P_{1}\right)=\left(\frac{1}{2}, \infty\right)$. For $P_{1}=1.618033989$, then $h_{1,-1}^{-1}(1.618033989)=\mp \sqrt{1.618033989+1}= \pm 1.618033989= \pm P_{1}$. But

$+P_{1}=1.618033989$ is a fixed point of $h_{1,0}(x)$, and $-1.618033989 \notin w_{l o c}^{u}\left(P_{1}\right)$.

Now for $h_{1,-1}^{-1}(-1.618033989)=h_{1,-1}^{-2}(1.618033989)=\mp \sqrt{-1.618033989+1}$. So $=\mp \sqrt{-0.618033989} \notin \mathbb{R}$

$h_{1,-1}(x)=x^{2}-1$ has no homoclinic points to the fixed point $P_{1}$

\subsection{Homoclinic Orbits for $h_{a, b}(x) \in H$}

In this part we study the homoclinic orbits for the family $H=\left\{h_{a, b}(x)=a x^{2}+b: a>0, b \in \mathbb{R}\right\}$.

\subsubsection{Remark}

For $b>\frac{-2}{a}$, we proved in theorem (5.1.9), $h_{a, b}(x)$ has no homoclinic points, 
so $h_{a, b}(x)$ has no homoclinic orbits for the repelling fixed point $P_{1}$.

To study the homoclinic orbits of $h_{a, b}(x)$ for $b \leq \frac{-2}{a}$, we introduce the following theorems and lemmas.

\subsubsection{Lemma}

For $h_{a, b}(x) \in H$ with $b \leq \frac{-2}{a}$, if $q<c$ where $c \in \mathbb{R}$ is a constant and $q$ is a homoclinic point to $P_{1}$, then $P_{2}+\frac{1}{a}<c$.

\section{Proof:}

Let $b \leq \frac{-2}{a}$, then $\sqrt{1-4 a b} \geq 3$, which implies $P_{2}=\frac{1-\sqrt{1-4 a b}}{2 a} \leq \frac{-1}{a}$, thus $P_{2}+\frac{1}{a} \leq 0$.

Now since $q \in w_{l o c}^{u}\left(P_{1}\right)$, then $q>\frac{1}{2 a}>0$ (see proposition (3.1)). So it is clearly $P_{2}+\frac{1}{a}<q$, thus $P_{2}+\frac{1}{a}<c$.

\subsubsection{Lemma}

$\left(P_{1}+P_{2}+\frac{1}{a}\right) c-P_{1}\left(P_{2}+\frac{1}{a}\right)=\frac{2 c-b-P_{1}}{a}$, where $c \in \mathbb{R}$ is a constant.

Proof:

$$
\begin{aligned}
& \text { Since } P_{1}=\frac{1+\sqrt{1-4 a b}}{2 a} \text { and } P_{2}=\frac{1-\sqrt{1-4 a b}}{2 a} . \\
& \left(P_{1}+P_{2}+\frac{1}{a}\right) c-P_{1}\left(P_{2}+\frac{1}{a}\right) \\
& =\left(\frac{1+\sqrt{1-4 a b}}{2 a}+\frac{1-\sqrt{1-4 a b}}{2 a}+\frac{1}{a}\right) c-\left(\frac{1+\sqrt{1-4 a b}}{2 a}\right)\left(\frac{1-\sqrt{1-4 a b}}{2 a}+\frac{1}{a}\right) \\
& =\left(\frac{2}{2 a}+\frac{1}{a}\right) c-\left(\frac{1-(1-4 a b)}{4 a^{2}}\right)-\left(\frac{\frac{1+\sqrt{1-4 a b}}{2 a}}{a}\right) \\
& =\left(\frac{2}{a}\right) c-\left(\frac{4 a b}{4 a^{2}}\right)-\left(\frac{P_{1}}{a}\right)=\left(\frac{2}{a}\right) c-\left(\frac{b}{a}\right)-\left(\frac{P_{1}}{a}\right)=\frac{2 c-b-P_{1}}{a}
\end{aligned}
$$

\subsubsection{Theorem}

Let $b \leq \frac{-2}{a}$ for $h_{a, b} \in H$. If $P_{2} \leq x \leq P_{1}$, then $\left\langle h_{a, b}^{-n}(x)\right\rangle$ is an increasing sequence.

\section{Proof:}

The first preimage of $h_{a, b}(x)$ is $h_{a, b}^{-1}(x)=\sqrt{\frac{x-b}{a}}$.

Claim: $h_{a, b}^{-1}(x)$ is increasing for $P_{2} \leq x \leq P_{1}$. To show this, let $h_{a, b}^{-1}(x) \geq x$, 
that is $\sqrt{\frac{x-b}{a}} \geq x$, thus $a x^{2}-x+b \leq 0$, there for $\left(x-P_{1}\right)\left(x-P_{2}\right) \leq 0$. Then either $x \leq P_{1}$ and $x \geq P_{2}$, or $x \geq P_{1}$ and $x \leq P_{2}$ (omitted because there is no intersection). Hence $h_{a, b}^{-1}(x)$ is increasing whenever $P_{2} \leq x \leq P_{1}$. Now $h_{a, b}^{-1}\left(h_{a, b}^{-1}(x)\right) \geq h_{a, b}^{-1}(x)$. Which implies $h_{a, b}^{-2}(x) \geq h_{a, b}^{-1}(x), \quad\left(h_{a, b}^{-1}\right.$ is increasing). Thus, we have $h_{a, b}^{-(n+1)}(x) \geq h_{a, b}^{-n}(x)$, for any $n \in N$.

\subsubsection{Theorem}

Let $b \leq \frac{-2}{a}$ and $h_{a, b}(x) \in H$. If $x \geq P_{1}$, then $\left\langle h_{a, b}^{-n}(x)\right\rangle$ is a decreasing sequence.

The proof is the same as the above theorem.

\subsubsection{Theorem}

For $h_{a, b}(x) \in H$ with $b \leq \frac{-2}{a}$, if $P_{2} \leq x \leq P_{1}$ then the upper bound of the increasing sequence of preimages of $X,\left\langle h_{a, b}^{-n}(x)\right\rangle$ is $P_{1}$.

\section{Proof:}

It is clear that $h_{a, b}^{-1}(x)=\mp \sqrt{\frac{x-b}{a}}$. We will prove $h_{a, b}^{-n}(x) \leq P_{1}$ by induction Since $x \leq P_{1}$, then $\frac{x-b}{a} \leq \frac{P_{1}-b}{a}$. Since $x \geq P_{2}$ and $P_{2}>b$ (because $h_{a, b}^{-1}\left(P_{2}\right)=\mp \sqrt{\frac{P_{2}-b}{a}}=\mp P_{2}$ so $h_{a, b}^{-1}\left(P_{2}\right)$ is undefined if $\left.P_{2}<b\right)$, then $x>b$. There for $\sqrt{\frac{x-b}{a}} \leq \sqrt{\frac{P_{1}-b}{a}}$. So, by lemma (4.1) then $h_{a, b}^{-1}(x) \leq P_{1}$

Now since $h_{a, b}\left(h_{a, b}^{-2}(x)\right)=h_{a, b}^{-1}(x)$, i.e. $a\left(h_{a, b}^{-2}(x)\right)^{2}+b=h_{a, b}^{-1}(x)$. Then by $\left(^{*}\right)$ we have $a\left(h_{a, b}^{-2}(x)\right)^{2}+b \leq P_{1}$, thus $h_{a, b}^{-2}(x) \leq \sqrt{\frac{P_{1}-b}{a}}$. Thus by lemma (4.1), then $h_{a, b}^{-2}(x) \leq P_{1}$.

Now assume that $h_{a, b}^{-(n-1)}(x) \leq P_{1}$ is true and we have to show $h_{a, b}^{-n}(x) \leq P_{1}$.

Since $a\left(h_{a, b}^{-n}(x)\right)^{2}+b=h_{a, b}^{-(n-1)}(x)$. Then, with our assumption

$h_{a, b}^{-(n-1)}(x) \leq P_{1}$, we get $a\left(h_{a, b}^{-n}(x)\right)^{2}+b \leq P_{1}$, thus $h_{a, b}^{-n}(x) \leq \sqrt{\frac{P_{1}-b}{a}}$. So by lemma (4.1), $h_{a, b}^{-n}(x) \leq P_{1}$.

\subsubsection{Theorem}

For $h_{a, b}(x) \in H$ with $b \leq \frac{-2}{a}$, if $x \geq P_{1}$ then the lower bound of the decreasing sequence $\left\langle h_{a, b}^{-n}(x)\right\rangle$ is $P_{1}$.

The proof is the same as the above theorem.

\subsubsection{Theorem}

If $b \leq \frac{-2}{a}, h_{a, b}(x) \in H$ and, $P_{2} \leq x \leq P_{1}$ then the supremum of the increasing 
sequence $\left\langle h_{a, b}^{-n}(x)\right\rangle$ is $P_{1} . \sup \left\{h_{a, b}^{-n}(x): n \geq 1\right\}=P_{1}$.

\section{Proof:}

The first preimage of $h_{a, b}(x)$ is $h_{a, b}^{-1}(x)=\sqrt{\frac{x-b}{a}}$.

If $\sup \left\{h_{a, b}^{-n}(x)\right\} \neq P_{1}$. Let $c<P_{1}$ and $\sup \left\{h_{a, b}^{-n}(x)\right\}=c$. There for $h_{a, b}^{-n}(x) \rightarrow c$. By lemma (5.2.2) $P_{2}+\frac{1}{a}<c$, there for $P_{2}+\frac{1}{a}<c<P_{1}$, which implies $P_{1}-c>0$.

Now let $\in=P_{1}-c$. Then $\exists k \in N$ such that $\left|h_{a, b}^{-n}(x)-c\right|<P_{1}-c, \forall n>k$, thus $\left|\left(a\left(h_{a, b}^{-(n+1)}(x)\right)^{2}+b\right)-c\right|<P_{1}-c$, which implies

$$
\begin{aligned}
& c-P_{1}<\left(a\left(h_{a, b}^{-(n+1)}(x)\right)^{2}+b\right)-c<P_{1}-c, \text { thus } \\
& \sqrt{\frac{2 c-b-P_{1}}{a}}<h_{a, b}^{-(n+1)}(x)<\sqrt{\frac{P_{1}-b}{a}} . \text { By lemma (4.1) and lemma (5.2.3), }
\end{aligned}
$$$$
\sqrt{\left(P_{1}+P_{2}+\frac{1}{a}\right) c-P_{1}\left(P_{2}+\frac{1}{a}\right)}<h_{a, b}^{-(n+1)}(x)<P_{1}
$$

But $c>P_{2}+\frac{1}{a}$ and $c<P_{1}$, which implies $\left(c-\left(P_{2}+\frac{1}{a}\right)\right)\left(c-P_{1}\right)<0$, thus $c^{2}-\left(P_{1}+P_{2}+\frac{1}{a}\right) c+P_{1}\left(P_{2}+\frac{1}{a}\right)<0$, hence $c^{2}<\left(P_{1}+P_{2}+\frac{1}{a}\right) c-P_{1}\left(P_{2}+\frac{1}{a}\right)$, there for $c<\sqrt{\left(P_{1}+P_{2}+\frac{1}{a}\right) c-P_{1}\left(P_{2}+\frac{1}{a}\right)}$. Thus by $\left(^{*}\right), \quad c<h_{a, b}^{-(n+1)}(x)<P_{1}$ which is a contradiction with $\sup \left\{h_{a, b}^{-n}(x)\right\}=c$. Thus $\sup \left\{h_{a, b}^{-n}(x)\right\}=P_{1}$.

\subsubsection{Theorem}

For $b \leq \frac{-2}{a}$ of the functions $h_{a, b}(x) \in H$ and $x \geq P_{1}$ then the infimum of a decreasing sequence $\left\langle h_{a, b}^{-n}(x)\right\rangle$ is $P_{1}$. inf $\left\{h_{a, b}^{-n}(x): n \geq 1\right\}=P_{1}$.

The proof is the same as the above theorem.

Finally, we introduce the main theorem in this section.

\subsubsection{Theorem}

For $h_{a, b}(x)=a x^{2}+b$ with $b \leq \frac{-2}{a}$ and $x \in \mathbb{R}$, the homoclinic orbist of the (first) homoclinic points $q_{2,1}$ and $q_{1,1}$ are $O\left(q_{2,1}\right)=\left\{P_{1},-P_{1}, q_{1,1}, q_{2,1}, \cdots, P_{1}\right\}$

$$
\begin{aligned}
& \text { for } \frac{-(5+2 \sqrt{5})}{4 a} \leq b \leq \frac{-2}{a} \text { and } O\left(q_{1,1}\right)=\left\{P_{1},-P_{1}, q_{1,1}, \cdots, P_{1}\right\} \text { for } \\
& b<\frac{-(5+2 \sqrt{5})}{4 a} \text {. }
\end{aligned}
$$

Proof:

Since $P_{1}=\frac{1+\sqrt{1-4 a b}}{2 a}$. Then 
1) By Remark (2.1), for $b<\frac{1}{4 a}, h_{a, b}^{\prime}\left(P_{1}\right)>1$.

2) By theorem (5.1.8), $h_{a, b}^{3}\left(q_{2,1}\right)=P_{1}$ for $\frac{-(5+2 \sqrt{5})}{4 a} \leq b \leq \frac{-2}{a}$ and $h_{a, b}^{2}\left(q_{1,1}\right)=P_{1}$ for $b<\frac{-(5+2 \sqrt{5})}{4 a}$ (see Figure 3 ). So $h_{a, b}^{n}\left(q_{2,1}\right)=P_{1}$ for $n \geq 3, h_{a, b}^{n}\left(q_{1,1}\right)=P_{1}$ for $n \geq 2$.

3) By theorems (5.2.4) and (5.2.8), (resp. (5.2.5) and (5.2.9)), $\left\langle h_{a, b}^{-n}\left(q_{j, 1}\right)\right\rangle$ where

$j=1,2$ is an increasing with supremum (resp. decreasing with infimum) $P_{1}$. Thus, $h_{a, b}^{-n}\left(q_{j, 1}\right) \rightarrow P_{1}$. There for, 1,2 and 3 show that $q_{2,1}, q_{1,1}$ are the homoclinic points for $P_{1}$ with the homoclinic orbits

$$
\begin{aligned}
& O\left(q_{2,1}\right)=\left\{P_{1},-P_{1}, q_{1,1}, q_{2,1}, \cdots, P_{1}\right\} \text { for } \frac{-(5+2 \sqrt{5})}{4 a} \leq b \leq \frac{-2}{a} \text { and } \\
& O\left(q_{1,1}\right)=\left\{P_{1},-P_{1}, q_{1,1}, \cdots, P_{1}\right\} \text { for } b<\frac{-(5+2 \sqrt{5})}{4 a} . \text { See Figure 3. }
\end{aligned}
$$

Following examples explain the cases for $b=\frac{-2}{a}$ and $b<\frac{-2}{a}$ respectively.

\subsubsection{Example}

For $h_{1,-2}(x)=x^{2}-2$, a homoclinic orbit of a homoclinic point $\sqrt{2}$ is $O(\sqrt{2})=\{2,-2,0, \sqrt{2}, \cdots, 2\}$.

\section{Solution:}

The forward orbit of $\sqrt{2}, \quad h_{1,-2}(\sqrt{2})=0, h_{1,-2}(0)=-2, h_{1,-2}(-2)=2$, thus $h_{1,-2}^{3}(\sqrt{2})=2$. So $h_{1,-2}^{n}(\sqrt{2})=2$ for $n \geq 3$.

The backward orbit of $\sqrt{2}$ is $h_{1,-2}^{-n}(\sqrt{2})=\{\sqrt{2}, \sqrt{\sqrt{2}+2}, \sqrt{\sqrt{\sqrt{2}+2}+2}, \cdots\}$.

To prove $h_{1,-2}^{-n}(\sqrt{2}) \rightarrow 2$. Consider the sequence $\left\langle h_{1,-2}^{-n}(\sqrt{2})\right\rangle=\left\langle a_{n}\right\rangle$.

$\left\langle a_{n}\right\rangle$ is an increasing sequence: It is easily shown that for $-1 \leq x \leq 2$ the function $\sqrt{x+2}$ is an increasing function. Hence $\cdots \geq h_{1,-2}^{-2}(x) \geq h_{1,-2}^{-1}(x) \geq x$, thus $\left\langle a_{n}\right\rangle$ is an increasing sequence.

Moreover for $-1 \leq x \leq 2$, by theorem (5.2.6) $h_{1,-2}^{-n}(x) \leq 2, \forall n$ (i.e. 2 is an upper bound for $\left.\left\langle a_{n}\right\rangle\right)$. Thus to show that $\left\langle h_{1,-2}^{-n}(x)\right\rangle$ converges to 2 , it is enough prove that $2=\sup \left\{\left\langle a_{n}\right\rangle\right\}$. If $2 \neq \sup \left\{\left\langle a_{n}\right\rangle\right\}$, let $c<2$ and $c=\sup \left\{\left\langle a_{n}\right\rangle\right\}$. By lemma (5.2.2) then $0<c<2$, since $\left\langle a_{n}\right\rangle$ is an increasing sequence and $c=\sup \left\{a_{n}: n \in N\right\}$, then $a_{n} \rightarrow c$. Now, since $0<c<2$, then $2-c>0$. Let $\in=2-c$.

Then $\exists k \in N$ such that $\left|a_{n}-c\right|<2-c, \forall n>k$.

Since the iteration of this sequence is $a_{n}=a_{n+1}^{2}-2$, thus $\left|\left(a_{n+1}^{2}-2\right)-c\right|<2-c$, which implies $2 c<a_{n+1}^{2}<4$, then $\sqrt{2 c}<a_{n+1}<2$

But $c>0$ and $c<2$ which implies $c(c-2)<0$, therefor $c<\sqrt{2 c}$. Thus by $\left.{ }^{*}\right), \quad c<a_{n+1}<2$ which is a contradiction with $c=\sup \left\{A\left\langle a_{n}\right\rangle\right\}$. Thus 
$2=\sup \left\{A\left\langle a_{n}\right\rangle\right\}$, and $a_{n} \rightarrow 2$. So $O(\sqrt{2})=\{2,-2,0, \sqrt{2}, \cdots, 2\}$ is a homoclinic orbit of a homoclinic point $\sqrt{2}$ for $h_{1,-2}(x)$.

\subsubsection{Example}

For $h_{1,-6}(x)=x^{2}-6$, a homoclinic orbit of a homoclinic point $\sqrt{3}$ is

$$
O(\sqrt{3})=\{3,-3, \sqrt{3}, \cdots, 3\} \text {. }
$$

\section{Solution:}

The forward orbit of $\sqrt{3}, \quad h_{1,-6}(\sqrt{3})=-3, h_{1,-6}(-3)=h_{1,-6}^{2}(\sqrt{3})=3$. So $h_{1,-6}^{n}(\sqrt{3})=3$ for $n \geq 2$.

The backward orbit of $\sqrt{3}$ is $h_{1,-6}^{-n}(\sqrt{3})=\{\sqrt{3}, \sqrt{\sqrt{3}+6}, \sqrt{\sqrt{\sqrt{3}+6}+6}, \cdots\}$.

To prove $h_{1,-6}^{-n}(\sqrt{3}) \rightarrow 3$. Consider the sequence $\left\langle h_{1,-6}^{-n}(\sqrt{3})\right\rangle=\left\langle a_{n}\right\rangle$.

$\left\langle a_{n}\right\rangle$ is an increasing sequence: It is easily shown that for $-2 \leq x \leq 3$ the function $\sqrt{x+6}$ is an increasing function. Hence $\cdots \geq h_{1,-6}^{-2}(x) \geq h_{1,-6}^{-1}(x) \geq x$, thus $\left\langle a_{n}\right\rangle$ is an increasing sequence.

Moreover, for $-2 \leq x \leq 3$, by theorem (5.2.6) $h_{1,-6}^{-n}(x) \leq 3, \forall n$ (i.e. 3 is an upper bound for $\left.\left\langle a_{n}\right\rangle\right)$. Thus to show that $\left\langle h_{1,-6}^{-n}(x)\right\rangle$ converges to 3 , it is enough prove that $3=\sup \left\{\left\langle a_{n}\right\rangle\right\}$. If $3 \neq \sup \left\{\left\langle a_{n}\right\rangle\right\}$, let $c<3$ and

$c=\sup \left\{\left\langle a_{n}\right\rangle\right\}$. By lemma (5.2.2) then $-1<c<3$, since $\left\langle a_{n}\right\rangle$ is an increasing sequence and $c=\sup \left\{a_{n}: n \in N\right\}$, then $a_{n} \rightarrow c$. Now, since $-1<c<3$, then $3-c>0$. Let $\in=3-c$.

Then $\exists k \in N$ such that $\left|a_{n}-c\right|<3-c, \forall n>k$.

Since the iteration of this sequence is $a_{n}=a_{n+1}^{2}-6$, thus $\left|\left(a_{n+1}^{2}-6\right)-c\right|<3-c$, which implies $2 c+3<a_{n+1}^{2}<9$, then $\sqrt{2 c+3}<a_{n+1}<3$

But $c>-1$ and $c<3$ which implies $(c+1)(c-3)<0$, there for $c<\sqrt{2 c+3}$. Thus by $\left(^{*}\right), c<a_{n+1}<3$ which is a contradiction with $c=\sup \left\{\left\langle a_{n}\right\rangle\right\}$. Thus $3=\sup \left\{\left\langle a_{n}\right\rangle\right\}$, and $a_{n} \rightarrow 3$. So $O(\sqrt{3})=\{3,-3, \sqrt{3}, \cdots, 3\}$ is a homoclinic orbit of a homoclinic point $\sqrt{3}$ for $h_{1,-6}(x)$.

\section{Conflicts of Interest}

The authors declare no conflicts of interest regarding the publication of this paper.

\section{References}

[1] Lord, G.J., Champneys, A.R. and Hunt, G.W. (1999) Computation of Homoclinic or-Bits in Partial Differential Equations: An Application to Cylindrical Shell Buckling. SIAM Journal on Scientific Computing, 21, 591-619. https://doi.org/10.1137/S1064827597321647

[2] Poincaré, H. (2017) The Three-Body Problem and the Equations of Dynamics: Poincaré's Foundational Work on Dynamical Systems Theory, Volume 443. Springer, Berlin. https://doi.org/10.1007/978-3-319-52899-1

[3] Smale, S. (1980) The Mathematics of Time. Springer, Berlin. https://doi.org/10.1007/978-1-4613-8101-3 
[4] Devaney, R. (2018) An Introduction to Chaotic Dynamical Systems. CRC Press, London. https://doi.org/10.4324/9780429502309

[5] Block, L.S. and Coppel, W.A. (2006) Dynamics in One Dimension. Springer, Berlin. https://www.springer.com/gp/book/9783540553090

[6] Gardini, L. (1994) Homoclinic Bifurcations in n-Dimensional Endomorphisms, Due to Expanding Periodic Points. Nonlinear Analysis. Theory, Methods and Applications, 23, 1039-1089. https://doi.org/10.1016/0362-546X(94)90198-8

[7] Marotto, F.R. and Fr, M. (1978) Snap-Back Repellers Imply Chaos in RN. https://doi.org/10.1016/0022-247X(78)90115-4

[8] Marotto, F.R. (2005) On Redefining a Snap-Back Repeller. Chaos, Solitons \& Fractals, 25, 25-28. https://doi.org/10.1016/j.chaos.2004.10.003

[9] Avrutin, V., Schenke, B. and Gardini, L. (2015) Calculation of Homoclinic and Hete-Roclinic Orbits in 1D Maps. Communications in Nonlinear Science and Numerical Simulation, 22, 1201-1214. https://doi.org/10.1016/j.cnsns.2014.07.008

[10] Onozaki, T. (2018) Nonlinearity, Bounded Rationality, and Heterogeneity. https://doi.org/10.1007/978-4-431-54971-0

[11] Chen, G. and Huang, Y. (2011) Chaotic Maps: Dynamics, Fractals, and Rapid Fluctuations. Synthesis Lectures on Mathematical Statistics, 4, 1-241. https://doi.org/10.2200/S00373ED1V01Y201107MAS011

[12] Laura, G., Viktor, A., Iryna, S. and Fabio, T. (2019) Continuous and Discontinuous Piecewise-Smooth One-Dimensional Maps: Invariant Sets and Bifurcation Structures, Volume 95. World Scientific, Singapore. https://lccn.loc.gov/2019017217 